\title{
Offene distale Humerusfraktur: Osteosynthese, Arthrodese oder Prothese?
}

\author{
Michael Paul Hahn
}

Der zum Unfallzeitpunkt 34-jährige Patient wird am 2.8.2004 als Motorradfahrer von einem Pkw angefahren. Bei dem Sturz erleidet der Patient nachstehende Verletzungen:

1. Eine 2.-gradig offene distale Humerusfraktur links (Abb.1),

2. eine geschlossene Talusfraktur links und

3. eine geschlossene Radiusextensionsfraktur rechts.

Bei der Erstversorgung am Unfalltag werden alle Frakturen osteosynthetisch versorgt. Die Radiusfraktur wird mit einer volaren winkelstabilen Platte fixiert. Die Talusfraktur wird mit zwei Schrauben versorgt.

Die offene Verletzung des linken Ellenbogengelenks stellt den Operateur vor ein schwieriges Problem. Teilweise fehlen knöcherne Fragmente, die am Unfallort verblieben sind. Damit erscheint eine anatomische Rekonstruktion unmöglich. Der Operateur entscheidet sich in dieser Situation zur Resektion zertrümmerter Knochenabschnitte. So wird auch der Ellenhaken entfernt. Die verbliebenen großen Fragmente des distalen Humerus werden mit 2 Schrauben fusioniert. Dieser Knochenblock wird dann mit einer winkelstabilen Platte an den distalen Humerus adaptiert. Bei reseziertem Olekranon wird die Platte um $90^{\circ}$ gebogen und anschließend an der Elle mit drei winkelstabilen Schrauben verankert. Damit endet die Osteosynthese dieser Fraktur in einer Arthrodese $[3,4]$.

Die postoperativen Bilder zeigen in beiden Ebenen keine ausreichende Adaptation des distalen Knochenblocks an den Humerusschaft. Die Arthrodese ist lediglich durch die Platte gesichert. Es

OP-JOURNAL 2008; 24: 204-205

(c) Georg Thieme Verlag KG Stuttgart • New York DOI 10.1055/s-2008-1039124

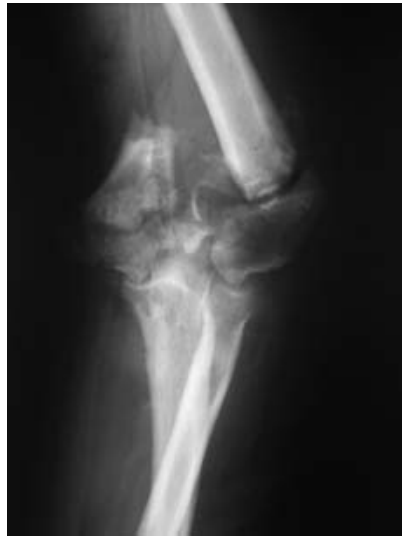

Abb. 1 Unfallbild linker Ellenbogen mit 2.-gradig offener distaler Humerustrümmerfraktur vom 2.8.2004.

fehlt eine knöcherne Verbindung zwischen Ulna und Humerus (Abb. 2).

Aufgrund der verbliebenen relativen Instabilität im Ellbogenbereich wird die Arthrodese am 3. postoperativen Tag durch einen Fixateur externe ergänzt (Abb. 3). Jetzt ist die Konstruktion stabil, weiterhin besteht ein großer Defekt zwischen Ulna und Humerus.

Nach eingehender Aufklärung des Patienten über mögliche Chancen und Risiken entschließt man sich zum Verfahrenswechsel $[1,2,5,6]$. Drei Wochen nach Unfall und Primärversorgung wird die Arthrodese des Ellbogengelenks wieder aufgelöst. Eine Coonrad-MorreyProthese wird einzementiert. Der Ellenhaken wird mit einem Knochenstück rekonstruiert (Abb. 4).

Sechs Wochen nach Prothesenimplantation zeigen sich reizfreie Weichteile mit primärer Wundheilung ohne Hinweis auf einen Infekt. Die Röntgenaufnahmen ergeben eine solide Verankerung der Ellenbogenprothese (Abb. 5).
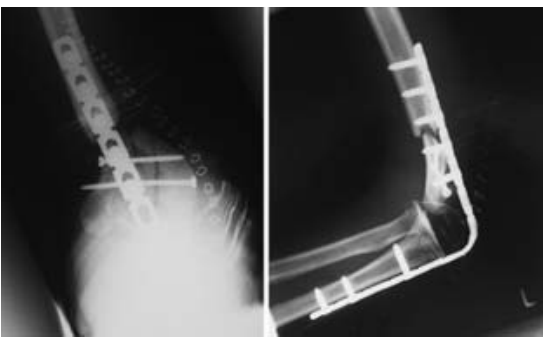

Abb. 2 Erstversorgung mit Arthrodese am Unfalltag.

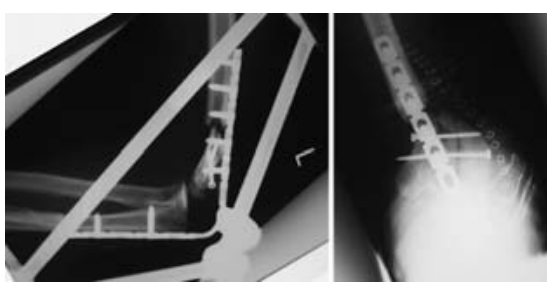

Abb. 3 Anlage Fixateur externe zur Sicherung der Arthrodese.

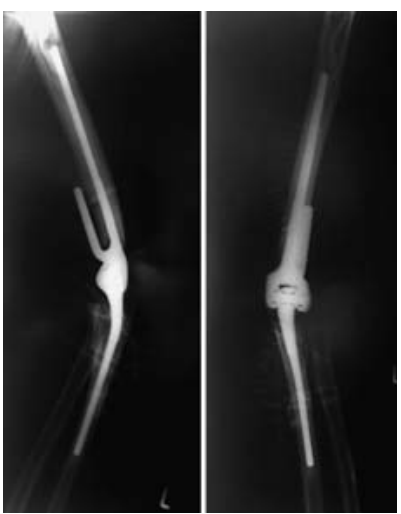

Abb.4 Implantation einer Ellenbogenprothese Typ CoonradMorrey 3 Wochen nach Unfall und Erstversorgung.

Auch im kurzfristigen Verlauf nach 3 Monaten ergeben sich keine Besonderheiten (Abb.6). Bei völlig reizlosen Weichteilen ist die Muskulatur noch verschmächtigt. Die Beweglichkeit beträgt für Streckung und Beugung $0 / 0 / 110^{\circ}$. 


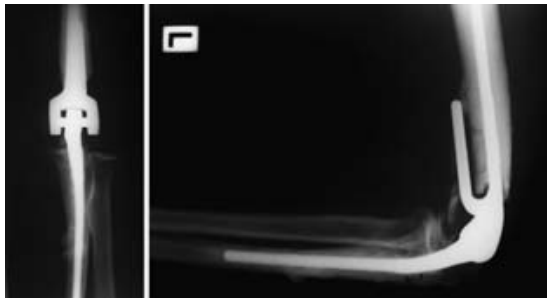

Abb. 5 Verlaufskontrolle 6 Wochen nach Prothesenimplantation.

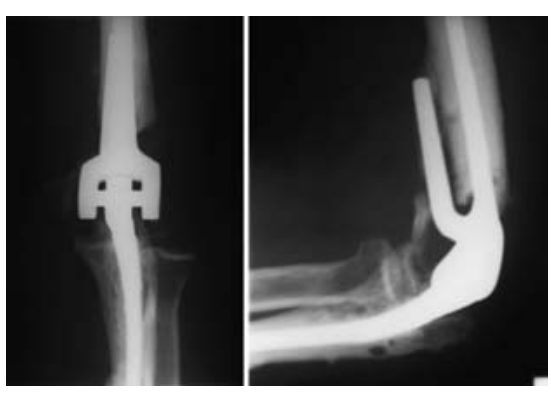

Abb. 6 Kontrolle 3 Monate nach Prothesenimplantation.

Die letzte Untersuchung des Patienten erfolgt 2 Jahre nach dem Unfall im Rahmen einer Begutachtung. $\mathrm{Zu}$ diesem Zeitpunkt ist der Patient beschwerdefrei. Die Beweglichkeit beträgt 0/0/120 . Mit diesem Bewegungsausmaß ist der Patient sehr zufrieden. Er ist weiterhin in der Lage, seiner Tätigkeit als Postzusteller nachzugehen.
Die Prothese weist in den Röntgenaufnahmen keine Lockerung auf. Die Aufnahmen stehen bei Erstellung des Manuskripts nicht zur Verfügung.

\section{Fazit}

Offene distale Humerustrümmerfrakturen stellen eine erhebliche Herausforderung dar. Wenn immer möglich sollte die Rekonstruktion versucht werden. Die Arthrodese des Gelenks ist für den jüngeren Patienten die schlechteste Alternative, da die Funktion des Ellenbogens nicht kompensiert werden kann [3]. In ausweglosen Fällen kann auch bei jüngeren Patienten mit guten Erfolgsaussichten eine Ellenbogenprothese implantiert werden [2]. Das Problem einer frühzeitigen Lockerung ist jedoch noch nicht gelöst.

\section{Literatur}

${ }^{1}$ Cobb TK, Morrey BF. Total elbow arthroplasty as primary treatment for distal humeral fractures in elderly patients. JBJS Am 1997; 79: 826-832

${ }^{2}$ Eichinger S, Forst R, Kindervater M. Indikationen und Alternativen der endoprothetischen Versorgung beim jüngeren Patienten. Orthopäde 2007; 36: 311-324

3 Hahn MP, Ostermann PAW, Richter D, Muhr G. Ellenbogenarthrodesen und ihre Alternativen. Orthopäde 1996; 25: 112-120

${ }^{4}$ McAuliffe JA, Burkhalter WE, Ouellette EA, Carneiro RS. Compression plate arthrodesis of the elbow. JBJS Br 1992; 74: 300-304
${ }^{5}$ Morrey BF. Fractures of the distal humerus: role of elbow replacement. Orthop Clin North Am 2000; 31: 145-154

${ }^{6}$ Müller LP, Kamineni S, Rommens PM, Morrey $B F$. Primary total elbow replacement for fractures of the distal humerus. Oper Orthop Traumatol 2005; 17: 119-142

Prof. Dr. med. Michael Paul Hahn Ärztlicher Direktor

Klinik für Unfallchirurgie und Orthopädie Klinikum Bremen Mitte gGmbH St.-Jürgen-Straße 1 28205 Bremen

E-Mail: michael-paul.hahn@ klinikum-bremen-mitte.de 